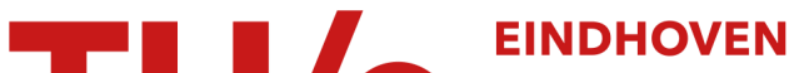 \\ UNIVERSITY OF \\ TECHNOLOGY
}

\section{Surface dynamics at photoactive liquid crystal polymer networks}

Citation for published version (APA):

Liu, D. (2019). Surface dynamics at photoactive liquid crystal polymer networks. Advanced Optical Materials, 7(16), [1900255]. https://doi.org/10.1002/adom.201900255

\section{Document license:}

TAVERNE

DOI:

10.1002/adom.201900255

Document status and date:

Published: 19/08/2019

\section{Document Version:}

Publisher's PDF, also known as Version of Record (includes final page, issue and volume numbers)

\section{Please check the document version of this publication:}

- A submitted manuscript is the version of the article upon submission and before peer-review. There can be important differences between the submitted version and the official published version of record. People interested in the research are advised to contact the author for the final version of the publication, or visit the $\mathrm{DOI}$ to the publisher's website.

- The final author version and the galley proof are versions of the publication after peer review.

- The final published version features the final layout of the paper including the volume, issue and page numbers.

Link to publication

\section{General rights}

Copyright and moral rights for the publications made accessible in the public portal are retained by the authors and/or other copyright owners and it is a condition of accessing publications that users recognise and abide by the legal requirements associated with these rights.

- Users may download and print one copy of any publication from the public portal for the purpose of private study or research.

- You may not further distribute the material or use it for any profit-making activity or commercial gain

- You may freely distribute the URL identifying the publication in the public portal.

If the publication is distributed under the terms of Article 25fa of the Dutch Copyright Act, indicated by the "Taverne" license above, please follow below link for the End User Agreement:

www.tue.nl/taverne

Take down policy

If you believe that this document breaches copyright please contact us at:

openaccess@tue.nl

providing details and we will investigate your claim. 


\title{
Surface Dynamics at Photoactive Liquid Crystal Polymer Networks
}

\author{
Danqing Liu
}

The field of advanced and responsive soft materials is at the edge of a new era. After several decades during which liquid crystals generated new functions for information displays and could solve many problems in emerging fields such as (tele)communications, this material system is being utilized to reach out to completely new application fields with functions that can take over biological actions (cell growth and manipulation), change the way materials, machines, or robots interact with humans (haptics), and modulate surface properties, e.g., tribology and wettability. This Progress Report concentrates on creating surface movement in liquid crystal networks with an emphasis on the light-responsive dynamic surface topographies that transfer from a flat to a predesigned corrugated state via light illumination, e.g., UV and visible light. Within this framework, the interaction between dynamic surfaces and the environment, such as controlled friction, wettability, and material transport, are illustrated. In addition, the light-induced thermal effect is discussed. The article concludes with the existing challenges and an outlook on the opportunities.

\section{Introduction}

The property by which materials produced by natural species often excel over man-made artificial materials is adaptivity. This is the case for shapes, whereby nature demonstrates numerous examples of animals that have the capability to morph, e.g., on the approach of a predator. However, adaptivity is often also present at the surface of living creatures. For example, mammals communicate through their skin by fluctuating its shape or reflecting piloerection to protect against predators. Moreover, the skin surface contributes to controlling body temperature by

Dr. D. Liu

Laboratory of Functional Organic Materials \& Devices (SFD)

Department of Chemical Engineering \& Chemistry

Eindhoven University of Technology

Den Dolech 2, 5612 AZ Eindhoven, The Netherlands

E-mail: d.liu1@tue.nl

Dr. D. Liu

Institute for Complex Molecular Systems (ICMS)

Eindhoven University of Technology

Den Dolech 2, 5612 AZ Eindhoven, The Netherlands

Dr. D. Liu

SCNU-TUE Joint Lab of Devices Integrated Responsive Materials (DIRM)

South China Normal University

No. 378, West Waihuan Road, Guangzhou Higher

Education Mega Center

Guangzhou 10006, China

The ORCID identification number(s) for the author(s) of this article can be found under https://doi.org/10.1002/adom.201900255.

DOI: 10.1002/adom.201900255 creating insulation under cold conditions and emitting evaporable liquid when it is warm. ${ }^{[1-3]}$ Peacock feathers, wings of certain butterflies, and plants exhibit color by means of structural color to communicate attraction or, inversely, repulsion. Often, the colors originate from periodic structures on or under a surface, thus benefiting from the interplay of light and diffractive effects.

A surface is the outermost layer of matter and therefore is primarily perceived by humans upon touch or vision. Surfaces provide perceptible information by not only shape, color, and/or reflective properties but also how the matter feels upon touch, e.g., smooth or rough. Welldocumented static structures have been reported for haptic applications. ${ }^{[4]}$ Studies have suggested that humans can distinguish changes in topographical dimensions down to nanometers. ${ }^{[5]}$ Additionally, a surface is the first contact between objects of matter, either of the same object or that of another origin. Surfaces facilitate man-machine interactions, ${ }^{[6]}$ either by touch (smooth, rough, heat conductive, etc.) or by vision (scattering, specular reflection, printed information, etc.). ${ }^{[7-9]}$ In relation to this function, tribological properties such as friction, stick, and adhesion coefficients also find application in the field of haptics, where robotic manipulation is of importance. In contrast to nature, the surface structures in modern man-made technology are often still static. Mimicking the lotus flower, surfaces with artificially formed micrometer-sized structures that are produced by lithography or micromachining have been made, and they indeed show improved cleaning under rainy conditions. However, one expands the surface function when these structures are made dynamic, rather than static, for example, the surface would be able to remove dirt under dry conditions, as well. In the case of topographies for haptic use, man's sensitivity could be enhanced when the corrugations are moving with a frequency tuned to the maximum sensitivity of humans. However, only a few publications have described dynamic structures to enhance human-surface interactions, which could even help visually disabled people. Moreover, in an advanced technology such as microfluidics, the low Reynolds number prohibits turbulent mixing of fluid components. Mixing can be induced by surface topographical structures in the microchannels to increase the contact area and the contact time between the reagents. The microfluidic functionality would be enhanced if the topography of its inner walls could be switched in a dynamic way, e.g., to decide on the exact location and time that switching 
should take place or to intensify mixing by high-frequency modulation of the surface structure.

For all these applications, adaptivity is key. In response to changes in the environment, the surface may change its mechanical and interactive properties to initiate a required function at the desired moment. In the class of soft materials, liquid crystals are known for their adaptive properties by responding to a variety of different triggers, such as temperature electrical and magnetic fields, light, and even motion and mechanical forces. To impart 'tangible' properties, the molecules are often chemically crosslinked to form a liquid crystal network (LCN) in the shape of a polymer film or coating. As such, liquid crystals have found their way to the display industry, where they enhance the optics and stability of the electrooptical elements of televisions, computers, and smart phones.

In this progress report, we elaborate the use of LCN technology to make photoresponsive surface topographies in which the surfaces, by illumination with light, can be switched from a flat state to a predesigned corrugated state or between two different corrugated states. We focus specifically on the most recent applications of dynamic surface topographies being the interfacial transport of matter (particles) to preset low-energy locations and the controlled migration and proliferation of living matter (cells) as stimulated by mechanical signals. The use of light to guide these processes has a number of benefits. It can be applied remotely and locally, precisely determining the location of action. It can also switch on and off in microseconds; this behavior is important not only in determining the moment of action but also deriving an oscillatory surface deformation, further enhancing motility at the interface.

To guide the readers in the discussion of present technological developments and future challenges, we begin the review by briefly introducing LCN technology, including the preparation, processing, and mechanical properties of materials. We detail the advantages of liquid crystal polymer networks in comparison to other reported responsive materials. We then review the underlying principles in creating surface topographies when exposed to light. The key features are the order parameter reduction, and under special conditions, a corresponding free volume generation. Based on these principles, new design options to create preset morphing surfaces in response to nonpatterned flood exposure to UV light will be elucidated. Recently developed interesting applications that are based on the interaction between dynamic surfaces and other matter will be discussed. We close the review by presenting the future challenges with respect to the development of materials and the range of their potential applications.

\section{Light-Responsive Liquid Crystal Networks}

\subsection{Liquid Crystal Network Technology}

In this progress report, we focus on LCNs that were developed in the 1980s by Broer and co-workers at Philips Research Laboratory. ${ }^{[10,11]}$ LCNs are polymers with well-controlled molecular positioning in all three dimensions. They are built up from liquid crystal monomers- the literature also refers to the monomers as reactive mesogens (RMs), which are liquid crystal

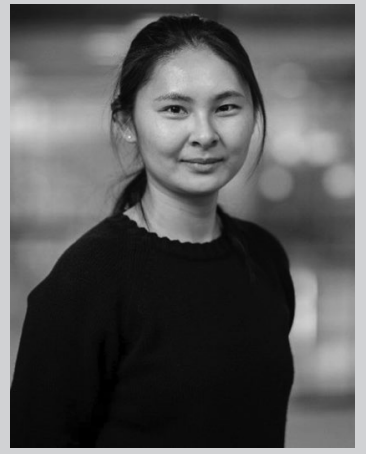

Danqing Liu received her bachelor degree from University of Electronic Science and Technology of China (Chengdu, CN) in 2006, the master degree from Delft University of Technology (Delft, Zuid-Holland, NL) in 2008, and completed her Ph.D. at Eindhoven University of Technology (Eindhoven, Noord-Brabant, NL) in 2013. Afterwards, she took a post-doc position at Eindhoven University of Technology, working as an ICMS research follow since 2016, and has been appointed to Assistant Professor in 2019. Her research interests include liquid crystals, soft robotics, interactive materials, and surface topographies.

molecules with polymerizable groups such as (meth)acrylates, epoxides, thiol-enes, or oxetanes, although most published work has involved acrylates by far. The polymerizable groups, one or two, can be attached to either end(s) or as a lateral side group. ${ }^{[12,13]}$ Because of their intrinsic anisotropic optical nature, the molecularly well-ordered LCs and LCNs exhibit unusual but very accurately adjustable and addressable optical, electrical, and mechanical properties. The electrooptics of low-molar-mass LCs have been widely explored by the modern liquid crystal display (LCD) industry, ${ }^{[14]}$ for example, electrically tuneable birefringence is used for most liquid crystal television displays. In specific configurations, LCNs are added to an active material to stabilize the liquid crystal alignment. Furthermore, LCN films are often added to high-end LCDs to improve the brightness, stability, and viewing angle or to add additional features such as a 3D viewing experience. ${ }^{[15]}$ In addition to their application in displays, in recent decades, scientists have investigated the use of liquid crystal technologies as stimuli-responsive materials for soft actuators and sensors by exploiting the (localized) control over the scalar order parameter in these well-ordered polymers. Compared with their hydrogel-based counterparts, LCNs possess a significant advantage in that they are a 'dry' technology for which their morphing is not restricted to the presence of a liquid, such as water. Additionally, actuation does not depend on a material's diffusion, which makes the switching of LCNs intrinsically faster. Furthermore, LCNs have an elastic modulus that is adjustable over a wide range, and they have good mechanical robustness.

The unique feature of LCNs is their capability to freeze in various molecular configurations. Currently, order is established in the monomeric state prior to polymerization, which is subsequently fixed during the (photo)polymerization process. ${ }^{[16,17]}$ Common examples include molecular alignments that comply with uniaxial, splay, bended, twisted nematic (TN), or tilted arrangements. The addition of chirality by modification of the LC monomer or by simply adding a chiral dopant induces helicoidal order, while polymerization in the Smectic phase preserves a layered molecular order in the polymer. There 
(a)

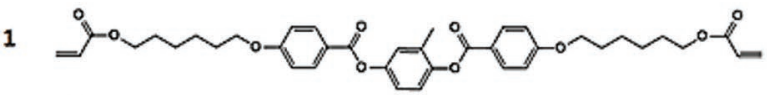

2

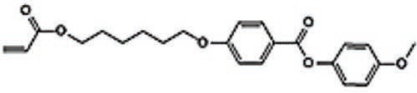

3

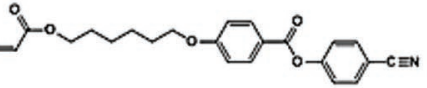

4

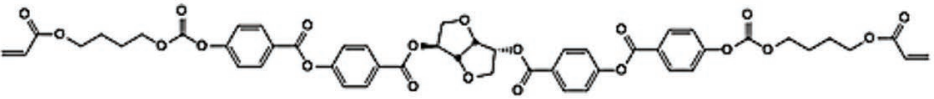

5

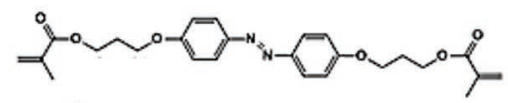

6

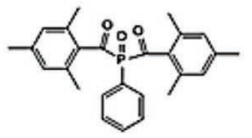

(b)

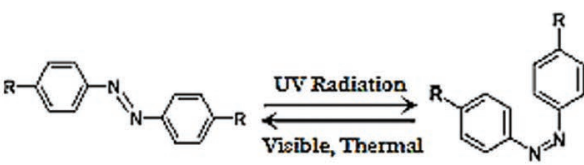

(c)

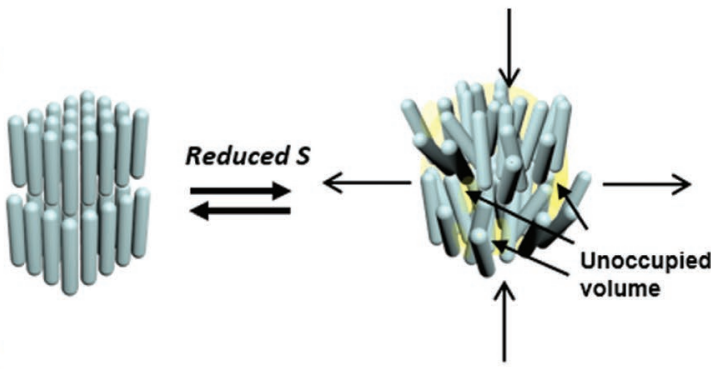

(d)

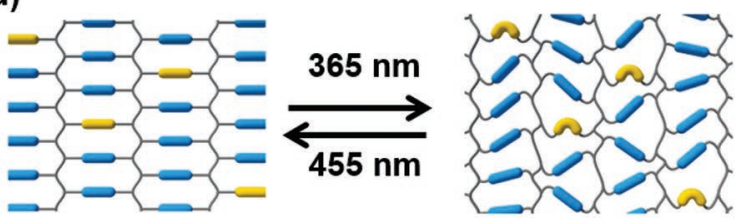

Figure 1. Liquid crystal networks. a) A typical composition of an LC monomer mixture to create a photosensitive chiral-nematic liquid crystal network. 1: diacrylate, 2: monoacrylate, 3: monoacrylate with a large dielectric anisotropy, 4: chiral dopant, 5: azobenzene, and 6: photoinitiator. b) The azobenzene molecule demonstrating its conformational change during UV exposure and its relaxation by blue light or temperature. c) A schematic representation of the anisotropic thermal expansion and free volume increase induced by the order parameter reduction. d) A schematic representation of the oscillating dynamics induced by the azobenzene trans-cis isomerization under combined UV and blue-light illumination. Reproduced with permission. [26] Copyright 2015, Springer.

are several techniques to control the molecular alignment, with most methods often being developed and matured by the LCD industry. For example, rubbed polymer (usually polyimide) surfaces provide a planar uniaxial alignment, whereas surfactanttreated surfaces provide alignment perpendicular to the surface (homeotropic alignment). Additionally, use can be made of external electric or magnetic fields or flow. Combinations of these alignment techniques are often used to create complex molecular architectures.

To optimize the properties both before and after polymerization, the monomeric precursors currently consist of a blend of LC mono- and diacrylates; for example, crystallization is suppressed by blending, which broadens the processing temperature range. The polymeric properties are affected by the crosslink density, which is adjusted by the proper ratio of mono- and difunctionalized LC monomers. Consequently, this ratio tunes the elastic modulus and the glass transition temperature $\left(T_{\mathrm{g}}\right)$ of the polymer coating or film. A typical mixture is given in Figure 1a. Upon polymerization, often initiated by light, the molecular alignment of the monomers is captured during the conversion to the polymer network. Normally, to ensure full conversion of the acrylate monomer, the sample is subjected to a postbake at an elevated temperature (typically, $\approx 120{ }^{\circ} \mathrm{C}$, 10-30 $\mathrm{min}$ ), where the polymerization process tends to cease when the glass transition is passed. The use of light to initiate the polymerization process allows for selective polymerization via photomasks. Therefore, the dimensions of the structures can be on the order of micrometers or larger. This method is a typical example of a structuring technology that combines selforganization (bottom-up) and lithography (top-down). Therefore, it is possible to integrate complex molecular alignments in a single film or coating when using LCNs.

\subsection{Scalar Order Parameter and Shape Changes}

Minor changes in the scalar order parameter of LCNs manifest themselves in sensible effects, such as anisotropic shape deformation, as illustrated in Figure 1c showing an expansion of the network orthogonal to the common orientation axis (director) and shrinkage parallel to that. ${ }^{[18,19]}$ This shows, for instance, that as the sample is heated from room temperature to, e.g., $150{ }^{\circ} \mathrm{C}$, the dimensions shrink parallel to the director, which results in a negative thermal expansion coefficient, and expand perpendicular to the director (positive thermal expansion, which is in general considerably higher than that of isotropic polymers) ${ }^{[20]}$ Under certain conditions, by matching the deformational oscillating dynamics to the network's viscoelastic properties, a dynamic free volume can be formed, which is in principal omnidirectional, but under substrate-constrained conditions, manifests itself in the $z$-direction perpendicular to the film surface. Not seldom, the triggered anisotropic expansion originates from a combination of both effects.

\subsubsection{Light-Induced Order Parameter Reduction and Free Volume Generation}

As discussed, for many applications, light is a preferred trigger, as it enables the remote control of a localized actuation in a noncontact manner. In general, covalently bonded azobenzene is used due to its photochromic and photomechanical properties. The molecule experiences a reversible trans-cis isomerization upon illumination with light at the proper wavelength (Figure 1b). By illumination with UV light, preferably in its absorption band $\approx 365 \mathrm{~nm}$, azobenzene undergoes a transition 
from its ground trans state to the thermally more unstable cis state. The reverse reaction to the trans state may occur thermally or by illumination with white light, where the absorption band of the cis state at $\approx 450 \mathrm{~nm}$ is addressed. The isomerization of azobenzene occurs with a geometrical change on the molecular level. From the trans (elongated) to the cis (bent) state, the molecular length changes from 9 to $5.5 \AA$. Based on this reaction, Seki et al. ${ }^{[21,22]}$ have demonstrated $2 \mathrm{D}$ dynamic surfaces that alternate their morphology in the $x-y$ plane of a Langmuir monolayer by attaching azobenzene to a polymer chain. ${ }^{[23]}$ Here, we concentrate on bringing surface topographies to a third dimension that protrudes from the LCN coating surface. While undergoing its transformation from the rod-like trans isomer, which easily coaligns with the surrounding mesogenic rods of the other monomeric units, to the bent cis isomer, in which the azobenzene moiety reduces the degree of order in the LCN. The bended configuration disturbs the alignment of the neighboring molecular units, and the length change of the azobenzene moiety in combination with the attachments at both of its ends exerts a considerable deforming force on the polymer main chains, which in turn brings the mesogenic rods further out of their initial close-to-parallel alignment. Consequently, this reduction in the scalar order parameter leads to an anisotropic deformation, as was discussed above for the thermal expansion.

Later, it was found that a change in the scalar order parameter could be accompanied by an extra volume increase (density decrease), see Figure 1c. ${ }^{[2,25]}$ Density measurements were performed on free-standing chiral-nematic films (composition as in Figure 1a) in the exposed and nonexposed states. In the nonexposed state, the density of the film measured at RT is $1.217 \mathrm{~g} \mathrm{~cm}^{-3}$ using a density column. Upon immersing this film in salt brine with a lower density, the LCN film sinks to the bottom. Upon exposure to UV light, the film starts to float to the surface of the salt brine indicating a density decrease. Switching off the light source causes it to sink again. From a series of these experiments with various salt concentrations, the authors estimate that the density of the exposed film is $1.106 \mathrm{~g} \mathrm{~cm}^{-3}$. This corresponds to a density reduction of $9.8 \%$. These measurements were performed by using a high-pressure mercury lamp emitting both UV and visible light, where the emission lines at 365,405 , and $436 \mathrm{~nm}$ appear to play dominant roles. Evidently, the combination of cis stimulating $365 \mathrm{~nm}$ and trans stimulating blue light provide a condition for the generation of a temporary free volume. The extra free volume increase can be further maximized by the careful choice of the ratio between the UV and blue light (Figure 1d) when the polymer network of well-ordered molecular mesogens is being subjected to an oscillatory molecular stress at a specific frequency. ${ }^{[26]}$

The expansion related to the anisotropic scalar order parameter explains the phenomena of free-standing thin films with complex director patterns, such as the bending of films with a splay organization, spiral formations, or the accordionlike deformations of films with (locally) twisted director patterns as well as even more-complex origami-types of deformations by well-designed director patterns. ${ }^{[27-36]}$ The volume increase in the free-standing film is not well recognized as it is generally isotropic and does not lead to bending deformations unless there is a gradient in the light absorption; however, then the response is normally overwhelmed by other deformation effects. Nevertheless, when the thin film is confined on a rigid substrate, the lateral deformation is largely prohibited, and the free volume formation becomes apparent, as the expansion can only occur at the coating surfaces manifesting in the formation of protrusions. This result becomes most clear in the local exposure of a homeotropic film. Based on arguments of the scalar order parameter, the film would shrink; however, because of the free volume formation, the film expands in the perpendicular direction forming a small hill rather than a valley. ${ }^{[37]}$ Further information on this experiment is described in section 2.2.2.

In the next section, we will elaborate various surface topographies and their corresponding underlying mechanisms based on geometric deformation, volume increase, or a combination of both. It should be noted at this point that upon light exposure, the thermal- and photochemical effects can never be fully separated. Often, the absorption of light by the azobenzene chromophore leads to an increase in temperature. In the case of LCNs applied as a coating on glass, the glass substrate acts as heat sink, and the sample does not absorb as much heat as a free-standing film does. However, when the molecules are introduced to a high-frequency oscillation, this translates to a very high localized thermal energy, as has been modeled by Clarck et al.[38]

\subsubsection{Formation of Dynamic Surface Topographies}

Based on Anisotropic Shear Stresses: A general assumption for the chosen coating configuration is that macroscopic anisotropic expansion does not take place due to the restriction of the rigid substrate, assuming also that flow is prohibited by the firm polymer network. However, this does not exclude the built up of shear stresses between the coating and the underlying, light- and temperature-inert glass substrate. An LCN film with a single director over the surface does not reveal remarkable changes in the surface pattern as long as wrinkling conditions are prevented. However, at the location where two director patterns meet, the lateral in-plane stresses find escape routes by expanding/shrinking into the third dimension. This phenomenon becomes even clearer when the substrate allows deformations. This has been demonstrated by Hendrikx et al. ${ }^{[39]}$ with a bilayer coating configuration, in which an LCN coating with orthogonal planar director patterns adheres to a soft compliant layer in between the LCN and the glass substrate. The compliant layer $(\approx 60 \mathrm{MPa})$ exhibits a substantially lower modulus compared with that of the hard glassy LCN top coating $(\approx 2 \mathrm{GPa}$ at room temperature). Upon actuation with UV, the built-in lateral stresses by the LCN due to the anisotropic deformation are dissipated at the interfacial orientational defect between the two directors resulting in an expansion at one side that is adjacently connected to a shrinkage at the other side of the defect. Typically, the formed protrusions have a height of tens of nanometers with a maximum modulation of $\approx 100 \mathrm{~nm}$. Interestingly, the modulation depth depends on the polarization direction of the UV light in relation to the local directors. Additionally, the structure could be made dynamic by rotation of the polarization of the LED light source. Sousa et al. ${ }^{[40]}$ have designed an LCN coating with the combination of an isotropic 
region and a chiral nematic area. Reducing the order parameter at elevated temperatures results in forming protrusions in the chiral nematic region with a height of a hundred nanometers. Babakhanova et al. ${ }^{[41]}$ have shown similar effects with an LCN coating on glass that exhibits a well-controlled director pattern of radially aligned elements containing a regular array of +1 and -1 defects. Changing the scalar order parameter by heating to the rubbery state above the glass transition, the LCN coating forms hills and valleys of tens of nanometers exactly at the locations of the defects where the directors coincide.

Based on Volume Generation: Under the conditions of heating above the glass transition temperature or of moderate exposure to UV light, the formation of a free volume is not sustained other than by classical mechanisms attributed to the mobility related to thermal expansion, which are also found in isotropic polymers. Additionally, when the molecular rods are deflecting, as the order parameter decrease indicates, the volume created between the rods is rapidly filled by the polymer's own entities, especially when the temperature rises far above the glass transition of the LCN. However, under the condition that one creates intermolecular voids in between the rods at sufficient frequency and that the network is not elastically very mobile, the voids can be sustained just long enough to create volume. These conditions are met when azobenzene is stimulated to a relatively high oscillation speed between trans and cis by exposing the sample to UV and blue light at the same time.

A good example demonstrating the existence of free volume in LCNs, next to the direct measurement of the density (vide supra), is the local exposure of a uniform homeotropic coating where the mesogenic units are aligned perpendicularly to the substrate surface. ${ }^{[3]}$ According to the classical anisotropic expansion arguments, the coating should contract at the exposed places, thus forming indentations, as illustrated in Figure 1a. In contrast, a homeotropically aligned LCN coating, when irradiated through a line mask, expands at the exposed locations. This is illustrated in Figure 2a,b showing the formation of $150 \mathrm{~nm}$ protrusions corresponding to a modulation of $2 \%$, as defined by the ratio between the height and the original film thickness. This result strongly suggests the volume formation is dominant in a constrained film, while the anisotropic expansion is less influential. However, at both edges of the protrusion, a small indentation is visible, pointing to some stresses and the influence of the rotation of the scalar order parameter.

For the experiment with homeotropic alignment, one should also consider that the azobenzene moiety is also oriented along the propagating direction of the light and is thus interacting less efficiently with the incoming light. To enhance the absorption of the incoming light, a planar molecular alignment is preferred. ${ }^{[42,43]}$ Here, a chiral-nematic (cholesteric) order with the helix axes oriented perpendicular to the film surface possesses some advantages. To introduce chirality, a chiral dopant (Figure 1a, molecule 4) is added and copolymerized with the LCN. Because of the planar rotational organization of the crosslinking molecular rods, cholesteric exhibit an in-plane symmetry. A reduction in the order parameter results in a positive expansion normal to the plane, whereas the expansion in the plane is close to zero, which then reduces the lateral stresses. Figure 2c,d shows the surface topographies formed by exposing the coating to a grid mask under UV light with the same intensity as for the homeotropically aligned LCN. The formed protrusions are $\approx 800 \mathrm{~nm}$ and correspond to a modulation of $8 \%$, which is considerably higher than that in the case of the homeotropic sample.

Combination of Anisotropic Thermal Expansion and Volume Increase: When LCN coatings consist of relatively small domains of different molecular orientations, under the right illumination conditions, the geometric deformations and volume expansion can work in concert to achieve a cumulative effect. Such a complex molecular architecture can be obtained through traditional lithographic approaches with multiple polymerization steps by use of photoalignment layers in which the various director patterns are recorded or, more conveniently, by molecular self-processes. Figure 2e presents the self-assembled fingerprint texture of a chiral LCN in which the helix axes are enforced to be parallel to the surface. The helix axes take this worm-like configuration, where the molecules upon their rotation are alternating parallel and perpendicular to the film surface. Fingerprint is fabricated by carefully balancing the helicoidally twisting force, which rotates the liquid crystal molecules, and the perpendicular anchoring force. When the fingerprints are exposed to broad spectrum UV light, the order parameter is reduced resulting in stresses whereby the planar area tends to expand in a plane perpendicular to the director and perpendicular to the surface. This action provides a positive stress component in the z-direction. For the homeotropic area the plane of expansion is parallel to the surface creating a negative stress component in the z-direction enforcing local shrinkage and forces acting on the neighboring planar area stimulating their expansion even more. In addition to this, the volume expansion is predominantly occurring in the planar area, as the dichroic nature of azobenzene favors UV absorption by the planaroriented chromophores. This sum of effects leads to considerable modulation depths, which for films $<10 \mu \mathrm{m}$ can reach values of $\approx 20 \%$, as shown in Figure $2 f^{\left[{ }^{[44]}\right.}$

Although, for the creation of fingerprint textures, the complex aligning processes are superfluous, and it remains not easy to balance the two distinct forces. A homeotropic film forms at too high a perpendicular anchoring force, and a planar alignment with rotated helix axes results from too dominant a rotational twisting force at small pitches. In practice, this means that the fingerprint only forms when the coating thickness is on the order of half the periodicity of the molecular helix. To circumvent this and to produce a dynamic surface via a more versatile coating process, light-responsive polydomain nematic LCN coatings were developed. ${ }^{[45]}$ In polydomain liquid crystals, molecules are aligned randomly in domains rather than over the entire coating area. Domain sizes on the order of 10-50 $\mu \mathrm{m}$ are controlled by dispersing a low concentration of small particles of a nondissolving fluorinated acrylate monomer in the monomer mixture. As with the fingerprint textures, there are regions with different directors; however, here they are distributed randomly over the surface. This arrangement gives a plural of effects whereby the planar regions tend to expand, the homeotropic to shrink, and the tilted to be less responsive. Additionally, on top of that, the many organizational defects respond on their own, as described earlier. The result is the formation of a spikelike surface with a topography of various heights. 
(a)

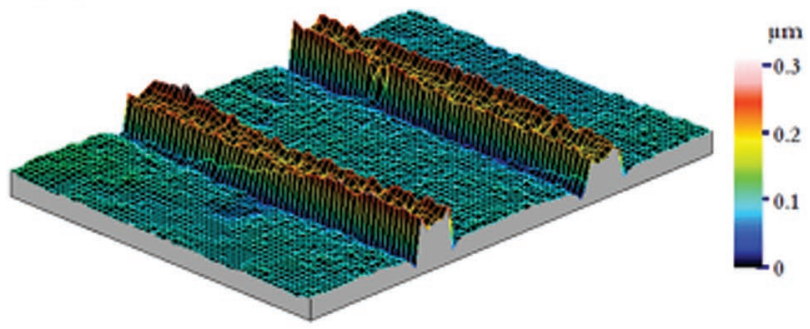

(c)

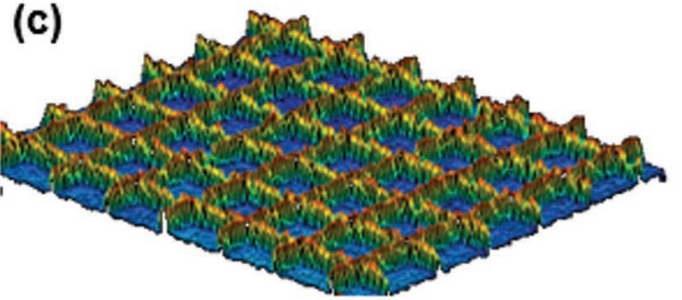

(e)
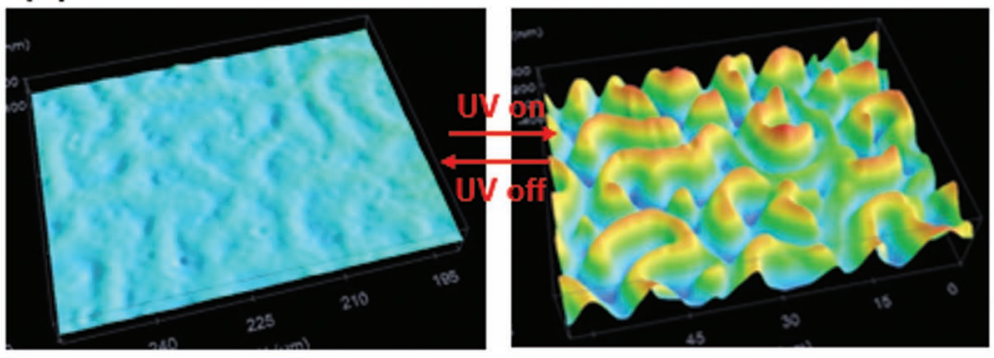

(b)

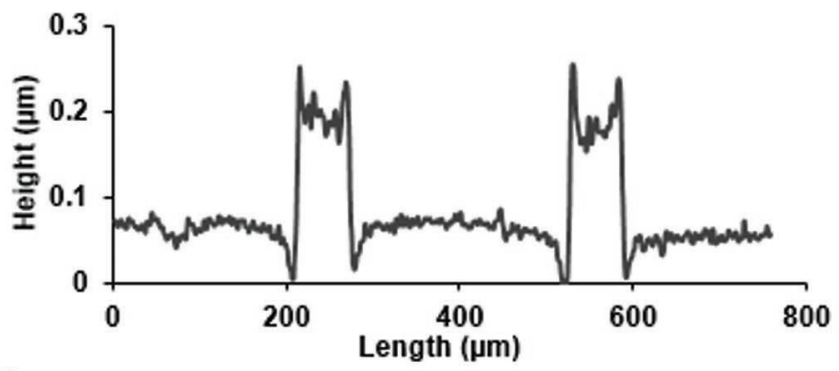

(d)

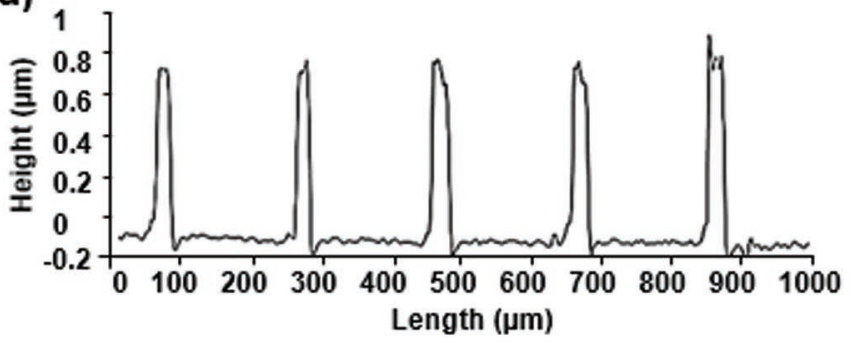

(g)

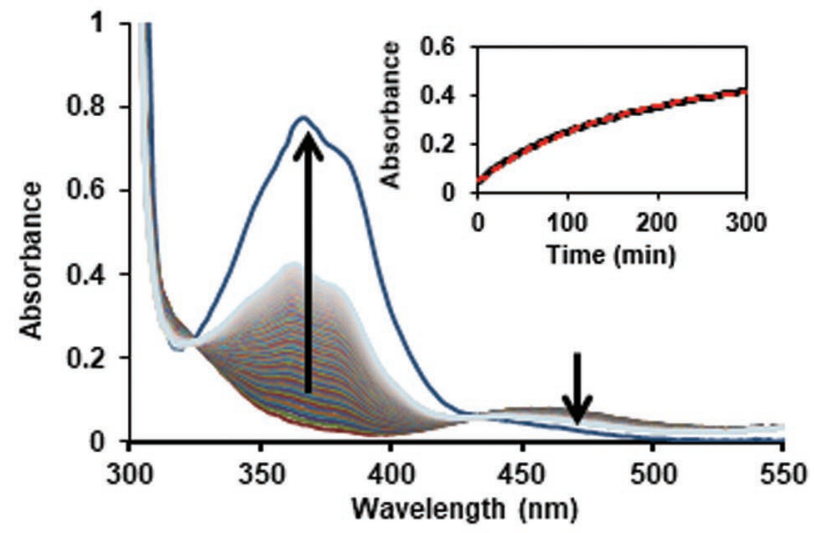

(f)

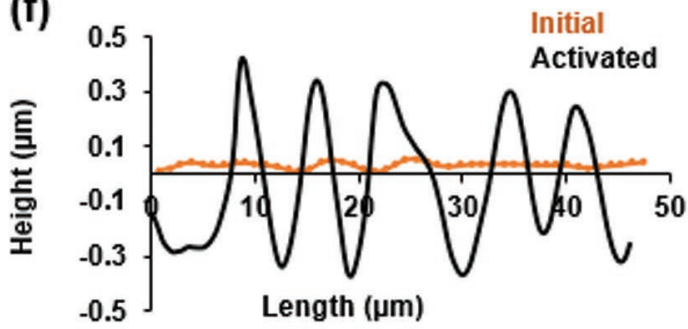

(h)

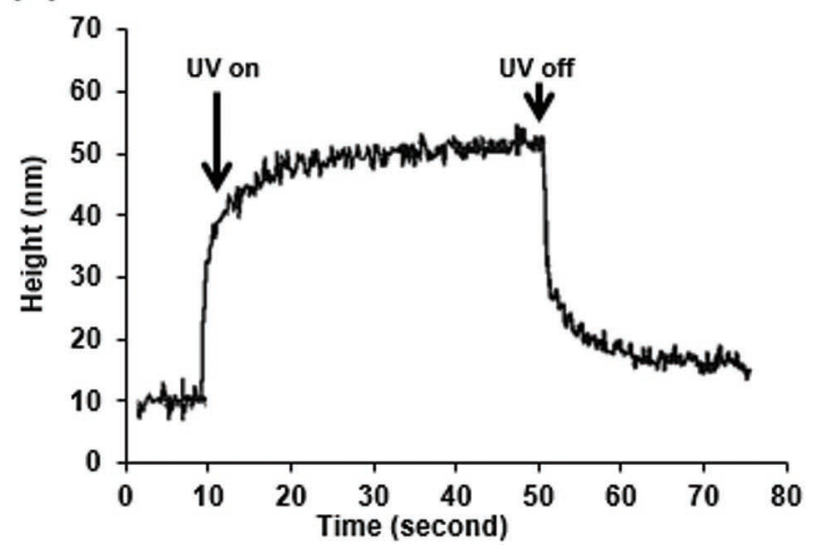

Figure 2. Dynamic surface topographies. a) A 3D view of the protrusions made by exposing the azobenzene-modified homeotropic film through a line mask (mercury lamp $300 \mathrm{~mW} \mathrm{~cm}{ }^{-2} ; 300 \mu \mathrm{m}$ pitch, $100 \mu \mathrm{m}$ opening). b) The corresponding 2D cross-section profile. c) A 3D image showing the surface topography formed in an azobenzene-modified chiral-nematic network by exposure through a grid mask $(225 \mu \mathrm{m}$ pitch, $25 \mu \mathrm{m}$ opening $)$ and d) the corresponding 2D cross-section profile. e) A 3D image of the fingerprint texture of a $4 \mu \mathrm{m}$ thick coating and f) the corresponding $2 \mathrm{D}$ cross-section profile. Reproduced with permission. ${ }^{[44]}$ Copyright 2014, Wiley-VCH. g) The mechanical kinetics of the LCN. h) The chemical relaxation of azobenzene in the dark. Reproduced with permission. ${ }^{[26]}$ Copyright 2015, Springer.

Actuation and Relaxation Dynamics: The formation of topographies upon exposure to UV light proceeds in general on a time scale of seconds. Figure $2 \mathrm{~g}$ shows a typical example. It has been found that during the actuation with light of an initially glassy $\mathrm{LCN}$, the glass transition temperature shifts to a lower value, whereas at the same time, the temperature somewhat increases. These coactions bring the coating into its viscoelastic region with the deformation occurring around the temperature where the tand of the LCN maximizes. This process takes time, and although the conversion of the trans isomer to the cis stationary state can be 
significantly faster, the process of surface deformation is mainly determined by the viscoelastic properties of the LCN.

The relaxation of the surface topography from its actuated state to its initial (close to) flat state is more complicated. This process very much depends on the principle that is chosen for the actuation. For instance, the structures that rely only on the change in the scalar order parameter due to trans-cis conversion of the azobenzene relax back with a rate corresponding to the thermal cis-trans back-conversion, which lasts hours for nonsubstituted azobenzene (Figure $2 \mathrm{~h}$ ). Typically, the half lifetime of cis-azobenzene in the LCN is measured to be $7 \mathrm{~h}$, as shown in Figure 2g. ${ }^{[26]}$ When the sample is heated or exposed to blue light, this process can be accelerated to proceed in seconds. In the case of a free-volume-dominated process, the formed molecular voids are thermodynamically very unstable, and the relaxation proceeds much faster, although the majority of the azobenzene molecules are still in their cis state. As shown in Figure $2 \mathrm{~g}$, the relaxation takes place typically within $10 \mathrm{~s}$ after switching off the UV exposure and is determined by the viscoelastic properties of the LCN.

\subsection{Light-Modulated Surface Friction}

In tribology science, many studies have referred to the importance of surface topography on friction and wear. ${ }^{[46]}$ Additionally, topographical elements affect adhesion, release, lubrication, and related contact phenomena. Controlling the friction at coating surfaces in a dynamic way by exposing the surface to UV light will impact the field of controlled tribology and is of interest to material scientists working in a variety of areas such as (micro, nano) robotics, friction and wear, human-machine interfaces, automobiles and avionics, and medical instruments. In a particular experiment, both the static and dynamic friction coefficients were measured between two surfaces with photosensible parallel ridges (Figure 2a) in their off (flat surface) and their activated (with protrusions) states. ${ }^{[47]}$ The measured static friction coefficient $\left(\mu_{\mathrm{s}}\right)$ of two flat coatings facing each other is 0.5 . When the coating is illuminated with UV light, the linear grating structures are formed, and consequently, the static friction force is subjected to change. When ridges are parallel to each other and sliding is promoted to occur in the direction perpendicular to the ridges, the static friction coefficient increases to 0.7 due to interlocking. In contrast, when the ridges are orthogonally aligned, the static friction coefficient decreases to 0.2 due to the reduction of the contact area. Comparably, the kinetic friction coefficient $\left(\mu_{\mathrm{k}}\right)$ between coating surfaces can be modulated by light in a similar manner. When the coatings are flat, $\mu_{\mathrm{k}}$ is 0.4 regardless of the direction of motion. Here, as with the static case, $\mu_{\mathrm{k}}$ changes when the ridges are switched "on" with an increase for the parallel orientation $\left(\mu_{\mathrm{k}}=0.6\right)$ and a decrease for the orthogonal orientation of the ridges $\left(\mu_{\mathrm{k}}=0.2\right)$ for perpendicular alignment. This result is nicely demonstrated for the latter case in Figure 3a, showing the friction force dropping during switching the UV lamp "on" and "off." The sliding force decreases dramatically within $5 \mathrm{~s}$ directly after the UV-light source is switched on. By stopping the light illumination, the friction force directly goes to the initial high value again within $10 \mathrm{~s}$.

An example of how the modulated friction can be utilized is shown in Figure 3b,c. Here, a set of two latent fingerprint (a)

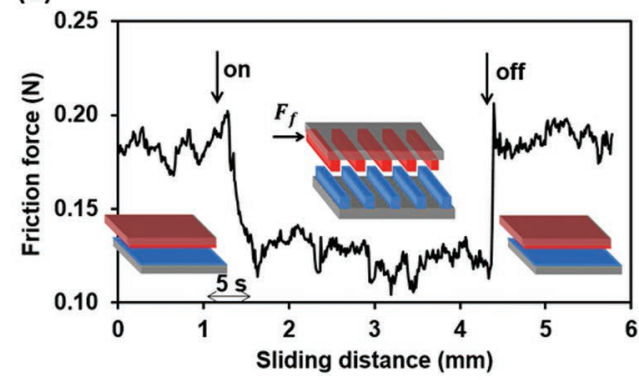

(c)

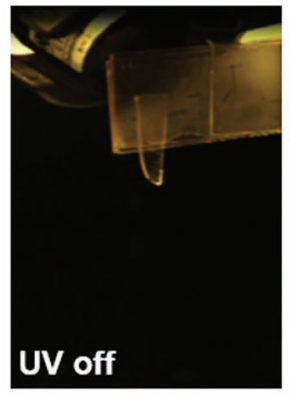

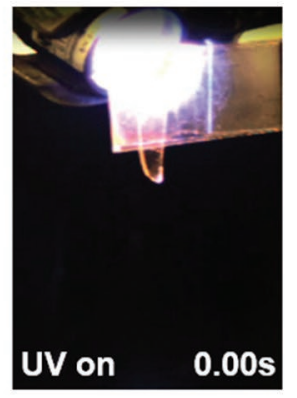

(b)
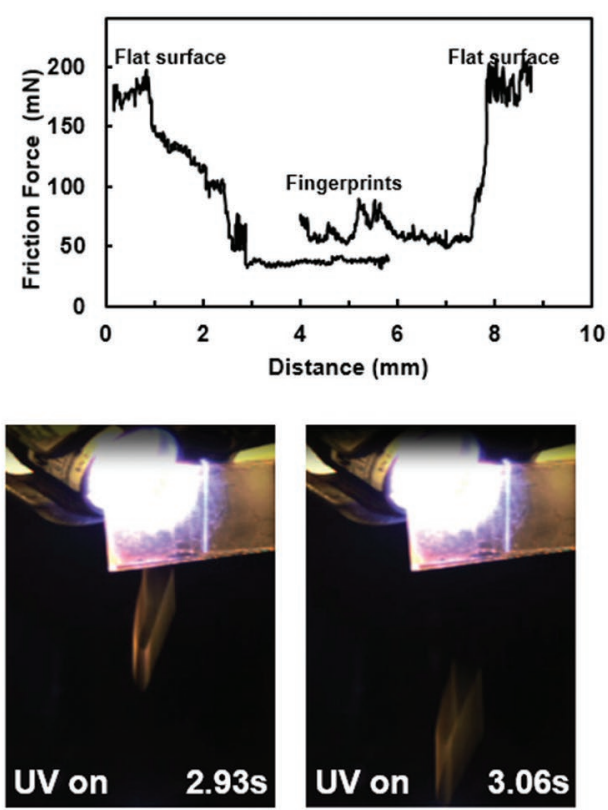

Figure 3. Dynamic friction modulated by light. a) The friction force between two glass plates sliding against to each other with their LCN coatings facing each other. During the measurement, the samples experience condition changes from the ambient (dark) to UV-light illumination to dark again ("on" and "off" switching of the UV-light source are indicated). In the measurement, linear grating structures are being formed with their orientation orthogonal to each other, as illustrated in the inset. b) The kinetic friction changes measured with and without UV-light exposure. c) The fingerprints formed in the LCNs assist releasing an object and snapshots of a gripper that releases an object upon UV illumination. Reproduced with permission. ${ }^{[44]} \mathrm{Copyright} 2014$, Wiley-VCH. 
textures capture a glass cargo, which is prevented from sliding by the high friction force when the contact area is maximized. When the fingerprint is switched "on" by the UV light, the contact area is reduced, the friction force is decreased, and the glass cargo is released. It is tempting to compare the switched artificial fingerprint with those of humans in which the ridgeshaped topography controls the grip and release of the objects. However, in this artificial case, the friction and grip are reduced, rather than increased. Grasping is retained through erasing the fingerprints by switching off the UV light. The underlying philosophy of this research is to assist robotic handling in picking up and releasing delicate objects. When robotic gripper tips are coated with soft elastic materials to protect the objects, they tend to stick to flat objects upon the aimed release. The addition of vibrational energy, in combination with lowfriction forces, provides a solution to this problem. In fact, the integration of human fingerprint coating on robotic finger tips is a good example of nature-inspired technology.

In the case of the polydomain coating, the activated surface topography is substantially more irregular and rough showing spike-like structures. Therefore, when two structures are brought in contact, they interlock, and the friction force increases. After switching off the UV light, the coatings relax back to the original flat state, the spikes are erased, and the friction force decreases to its original low value within $10 \mathrm{~s}$, corresponding to the measured kinetics for the disappearance of the surface structures.

\subsection{Tuneable Wettability Controlled by Light}

The wettability of a surface is an important interfacial property for many applications, varying from self-cleaning to water- or solvent-based manufacturing. The wettability of a solid surface is governed by two factors. One is surface energy, including its dispersive and polar contributions, which is an intrinsic prosperity of the material and can be adjusted chemically. The other factor is surface roughness in the form of surface topography. The control over wettability involves the modulation of either factor or even both factors. A typical example from nature that combines both factors is the lotus leaf. The lotus leaf is highly water-repellent because of its super-hydrophobic nature, as described in the introduction. The lotus leaf surface has an extremely small sliding angle, i.e., the minimum angle of a surface for which a droplet starts to slide off. The superhydrophobicity of the lotus leaf is due to the combination of a hydrophobic wax and hierarchical micro/nanostructures. This is not a unique property, as it is quite commonly observed in nature. Other plants, such as the paddle cactus and cane, and animals, including water striders and mosquito eyes, demonstrate similar surface features.

Making wettability switchable has attracted considerable attention in the scientific community due to the wide range of potential applications, such as in droplet manipulation, controllable drug delivery, controllable coating and printing processes, and self-cleaning surfaces. In most work, reversibility is established by switching the intrinsic hydrophobicity of the solid material. Here, we will discuss dynamic surface topographies that are employed to control the wettability. The main advantage is that the surface is less prone to (mechanical) damage since the surface topographies can be "switched off" at times in which it is not needed or when mechanical damage may occur.

The initial flat surface of the LCN as discussed shows moderate hydrophilicity with a water contact angle of $\approx 75^{\circ}$. When the topographic patterns (as shown in Figure 2c) are switched "on" by UV light, the contact angle increases to close to $100^{\circ}$, and the surface becomes more hydrophobic (Figure 4a). To
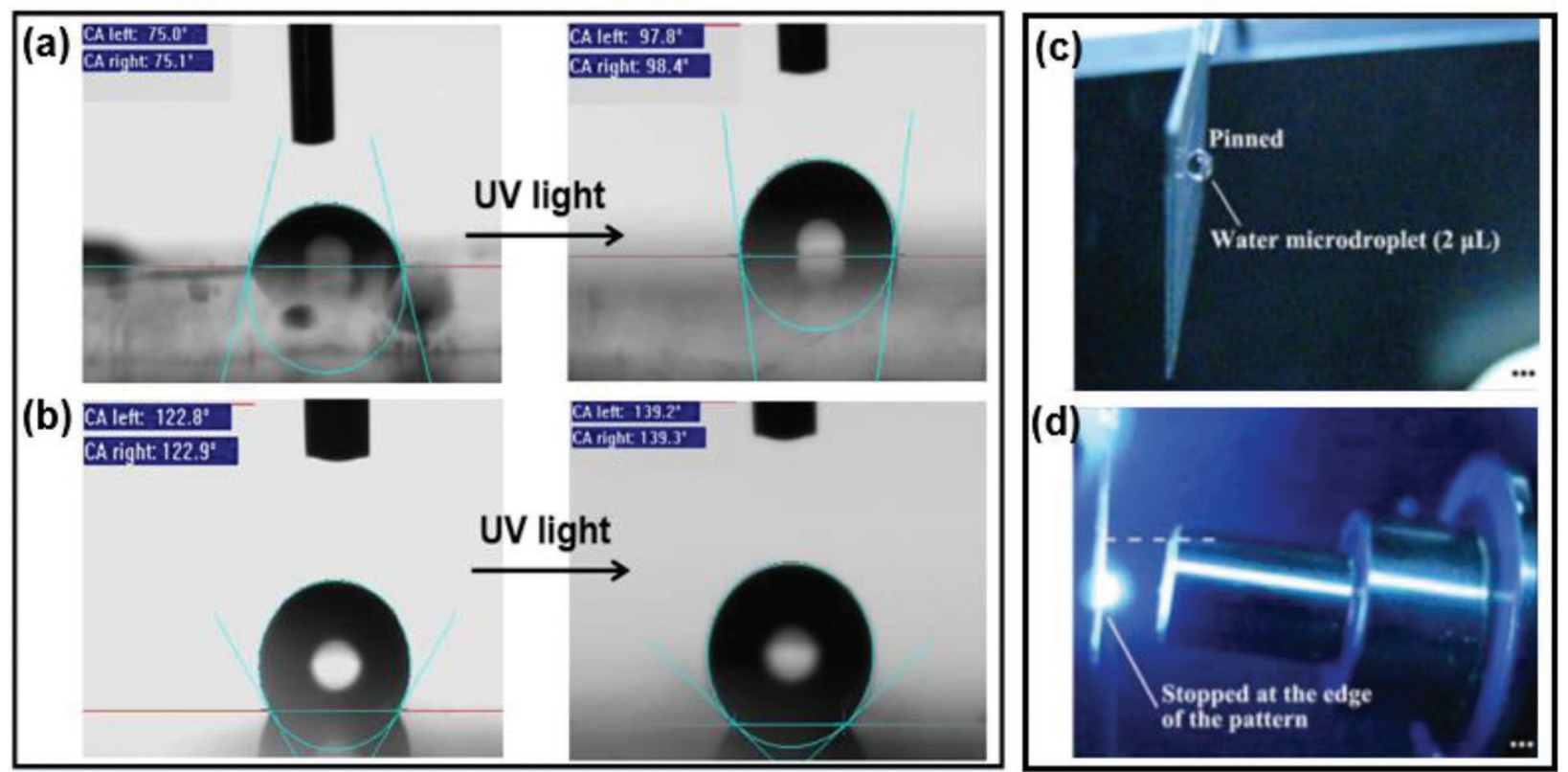

Figure 4. Light-modulated wettability of a coating surface. a) The surface is switched from hydrophilic to hydrophobic when it transforms from a flat to a corrugated state (the surface pattern is as shown in Figure 2c). b) A fluorinated-silane-modified coating surface is switched from a hydrophobic to super-hydrophobic state by means of the topography shown in Figure 2c. c) The water droplet is pinned at the coating surface after UV illumination. d) The adhesion force decreases during visible-light exposure, and the water droplet rolls off. Reproduced with permission. ${ }^{[49]} \mathrm{Copyright} 2011$, Wiley-VCH. 
further increase the hydrophobicity, the LCN coating surface is modified via silanization with fluorinated groups $(1 \mathrm{H}, 1 \mathrm{H}, 2 \mathrm{H}, 2 \mathrm{H}$-perfluoro-dodecyl-trichloro-silane). The contact angle measured for the flat state is $\approx 120^{\circ}$ (see Figure $4 \mathrm{~b}$ ). Upon UV illumination, the topographic textures are activated, and the surface becomes super-hydrophobic with a contact angle of $\approx 140^{\circ}$. This preliminary study ${ }^{[48]}$ proves the principle, however, a number of challenges need to be approached before this approach can be used as a switchable self-cleaning/tuneable surface. For instance, for wettability control, the lateral dimension of the topography needs to be downscaled to the micrometer or sub-micrometer level. Additionally, hierarchical substructures may be needed to achieve or improve the hydrophobicity. Creating secondary or superposed surface patterns involves not only enhancing the material resolution but also developing a dedicated fabrication strategy.

Yu et al. ${ }^{[49]}$ have developed an approach to dynamically control water adhesion through light illumination. In their method, an azopolymer is coated on a prestructured surface with microsized pillars that provide an initial hydrophobicity at the surface. The pillars are fabricated via a conventional lithographic process and are followed by chemical etching steps. Prior to UV actuation, the surface is hydrophobic with a contact angle of $140^{\circ}$. When exposed to UV light, both the surface chemical properties and the topographic roughness are modulated. On one hand, the surface polarity is changed due to the trans-cis isomerization of azobenzene in which the cis isomer has a considerably higher dipole moment than the trans isomer. On the other hand, the surface roughness increases slightly by the formation of secondary random textures in the nanometer range. By alternating UV- and visible-light irradiation, the hydrophobic adhesion is modulated. As shown in Figure 4c,d, a water droplet is pinned at the surface when illuminated with UV light for $5 \mathrm{~s}$. The water droplet adhesion force is measured as $80 \mu \mathrm{N}$. After $10 \mathrm{~s}$ of visible-light irradiation, the adhesion force decreases to $60 \mu \mathrm{N}$. Consequently, the water droplet rolls off.

\subsection{Dynamic Surface Topographies Interact with Matter}

The surface of cholesteric liquid crystals against air are often corrugated because of the Marangoni effect. In 2006, Feringa and co-workers showed that by embedding a molecular motor in a fingerprint chiral-nematic liquid crystal film, a rotational surface relief is generated under a continuous light illumination. ${ }^{[50,51]}$ This molecular motor is a light-responsive chiral molecule, which inverts its chirality under UV exposure, which modifies the pitch of the molecular helices and thereby rotates the surface undulations. Furthermore, this approach translates molecular phenomena into a macroscopic effect, and it also can exert forces demonstrated by the rotation of a glass rod that is brought in contact with the surface. Although this demonstration is relevant to showing the principle of upconverting molecular conformational effects to the macroscopic world and shows an optical application as diffraction gratings, ${ }^{[52]}$ its practical function is still limited because of the liquid nature of the liquid crystal. Therefore, in the next examples, we elaborate on the interaction between matter, e.g., the glass rod and the spheres, and the surface dynamics of solid LCN polymer coatings.
Manipulating matter at the LCN surface has been demonstrated by Babakhanova et al. ${ }^{[53]}$ Microparticles are transported and allocated to preset locations on the surface of an intermittently UV-exposed LCN surface sculpting and erasing topographic patterns. The topographies are set by patterned molecular alignment using a plasmonic metamask (PMM)-based photopatterning technique. On the initially close-to-flat surface, the particles are randomly distributed. Under illumination of the UV light, the trans-cis isomerization of copolymerized azobenzene molecules reduces the scaler order parameter of the LCN and activates the surface topographic patterns as set by director pattern. ${ }^{[41]}$ The particles are observed to follow the surface profile and move toward the valley of the topography, where they accumulate due to the gravitational forces. Upon erasing the surface topography through blue-light irradiation, the distribution of the particles becomes random, indicating the reversibility. These results are anticipated to be expanded to biological applications in which cell growth, migration, tissue formation, and even drug release are of importance, and this topic will be addressed in section 3.4 .

The above-described dynamic coating can move and place the particles; however, due to the symmetrical structures, directionality has not been addressed. The addition of a directional lateral component to the generated forces is capable of transporting matter at the surface. The most common way to induce directionality is through breaking symmetry and creating asymmetrical patterns, for instance, the ratchet structures. ${ }^{[54]}$ Static ratchets have been explored widely. However, to the best of our knowledge, switching a ratchet's surface "on" and "off" by light has not yet been demonstrated in LCNs, as it requires sophisticated designs and complex engineering. We expect this design to be developed in the future.

An alternative approach to transport matter is exploited through taking advantage of gravity by tilting the surface from its horizontal position. ${ }^{[55]}$ The continuous generation of surface undulations at the surface of LCNs has been demonstrated to successfully transport sand dust to the edge of the coating and to eventually slide them off. Although in this research, the surface dynamics are generated by an electric field, we believe the aimed effect presents a good example of showing the interaction between oscillating LCN surfaces and dust. Additionally, a similar effect can be created by light in an LCN with a preset fingerprint pattern. When activated with modulated light, the fingerprint inverts, and the initially higher homeotropic region descends to form valleys, while the originally lower area with planar alignment protrudes to become peaks (see Figure 5a). Sand particles with a dimension of $250-500 \mu \mathrm{m}$, significantly larger than the periodicity of the fingerprints, are brought on top of the fingerprint, and directly after switching on the trigger, $\approx 100 \%$ of the sand grains are removed within $1 \mathrm{~s}$. The significance of this study is that it demonstrates a dust mitigation technology. Most self-cleaning solutions are based on super-hydrophobic surfaces, which need water to clean. However, in many dry and dusty areas, the access to water is limited, and water-based self-cleaning approaches are not applicable. The LCN dynamic surface enables cleaning without the aid of water, where dust and debris are mechanically removed to the edges of the coating. Apparently, dust hazards are considered to be one of the most 
(a)

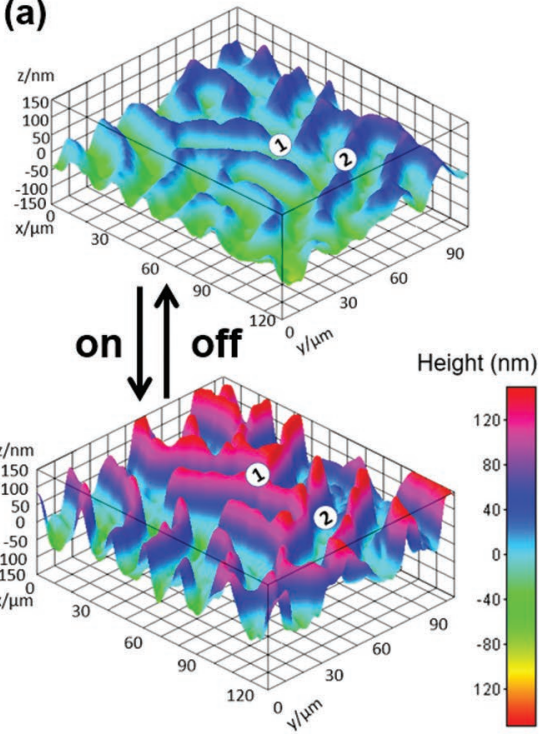

(b)

(d)
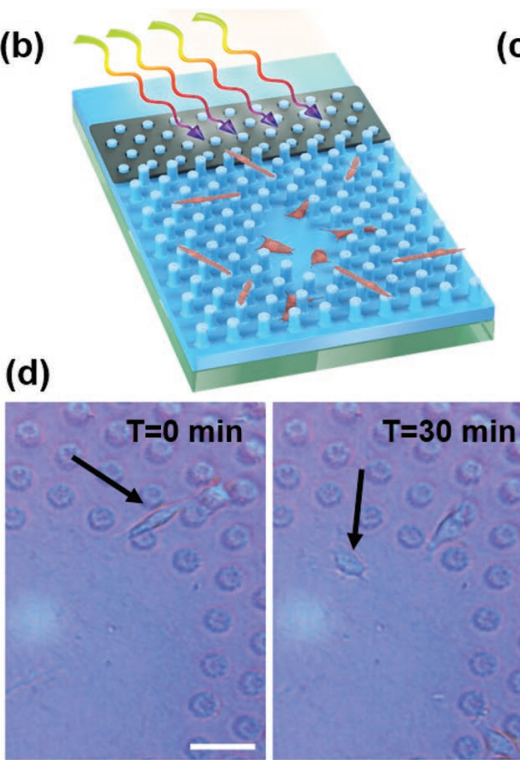

(c)
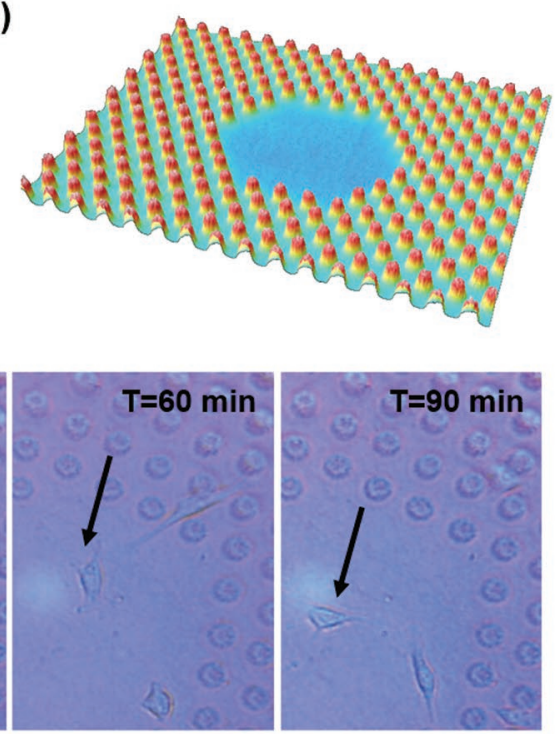

Figure 5. Dynamic surface topographies interact with matter. a) The fingerprint reverses the surface topography in which the initial peaks become valleys and the valleys become peaks. Reproduced with permission. ${ }^{[55]}$ Copyright 2017 , Wiley-VCH. b) An artistic rendition of controlling cell migration through dynamic surface topographies under the illumination of UV light. c) A 3D image of the formed surface topography, as measured by confocal microscopy. d) Snapshots of a cell migrating from a pillar area to a flat area. Reproduced with permission. ${ }^{[61]}$ Copyright 2017, Wiley- $\mathrm{VCH}$.

important problems to be resolved for Mars missions. NASA has been actively developing dust mitigation technologies to assist Mars missions since 2005. This technology will also solve the problem for solar farms placed in deserts where accumulated dust and sand block the sun light. Experiments performed by MIT have shown that one dust storm can cut power production by $40 \% \cdot{ }^{[56,57]}$

Next, using the dry-cleaning methodology, the surface structures can switch to the super-hydrophobic state after a modification with a fluorinated top layer and combine this action with oscillating surface dynamics. This duo makes the activated removal of wet dust possible. Humid dust adheres firmly to coating surfaces and has proven extremely difficult to remove due to the presence of capillary forces between the particles and the surface. The flexibility of combining both dry and wet self-cleaning principles allows for the coating to function under various conditions.

\subsection{Dynamic Surface Topographies Interact with Living Matter}

Controlling living matter in liquid crystals has been shown by a number of researchers, for example, Zhou et al. ${ }^{[5]}$ and Mushenheim et al., ${ }^{[59]}$ in which they place self-propelled particles, such as bacteria, in nematic liquid crystals. The particles possess a typical rod-like shape caused by the flagella that are attached to their body. At rest, the particles align well with the director of the surrounding liquid crystal molecules. When the self-propelling particles are active, they mutually influence the alignment of the liquid crystal in which the anisotropy of the fluid environment introduces a certain directionality to the bacteria; on the other hand, the motion of the bacteria alters the orientational order in the liquid crystals. ${ }^{[60]}$ These studies, however, are still performed in the liquid phase. We will here elaborate on the interaction between cells and solid glassy polymer coatings.

Koçer et al. ${ }^{[61]}$ have reported the manipulation of cell adhesion and migration by dynamic surface topographies in LCNs under UV-light exposure (Figure 5b). Light-responsive LCNs with chiral-nematic order are incubated and coated with serum proteins prior to the cell experiment to facilitate cell adhesion. The contact angle is then close to $90^{\circ}$ showing hydrophobicity, and the cell migration speed of NIH3T3 fibroblasts is $0.85 \mu \mathrm{m} \mathrm{min}{ }^{-1}$. When the coating is subjected to a flood of UV illumination, without the notable formation of a surface topography, the cell migration speed drops significantly to $0.32 \mu \mathrm{m} \mathrm{min}{ }^{-1}$. This result suggests that the motility of the cells can be switched from dynamic to moderately static (adhesion increase) by switching on the UV light. An atomic force microscopy measurement of the LCN surface reveals that the surface roughness changes from $\approx 10 \mathrm{~nm}$ in the dark to $\approx 18 \mathrm{~nm}$ during UV illumination, which could explain the increased adhesive interaction between the cells and coating surface. This observation is consistent with the literature reports, which claim that cells can sense small features down to $8 \mathrm{~nm} \cdot{ }^{[62]}$ It is interesting to note that in the foregoing experiment (described in Figure 4a, section 3.2), similar surface roughness is generated to control the water adhesion at the surface when the coating is exposed to UV light. Although the exact mechanism has not been elucidated, it represents a general effect of azobenzene-modified polymer networks and might be attributed to the geometric changes of azobenzene isomerization.

Creating larger surface structures in the same LCN coating, such as hexagonally arranged pillars by UV exposure through a photomask (presented in Figure $5 \mathrm{c}$ ), reveals that more than $80 \%$ of the cells tend to migrate from the pillar area to the flat area. In the flat region, the cells slow down and eventually become rested. Simultaneously, their morphology transfers 
from an elongated shape to a spread configuration, as shown in Figure 5d. These results suggest that the topographies of the surface influence cell morphology, which is known to affect cell motility. Koçer and co-authors have further discovered that the higher the formed pillar is, the faster the motion of the cells. When the height of the pillars increases from 0.3 to $0.5 \mu \mathrm{m}$, the corresponding cell migration speed increases dramatically from 0.23 to $0.87 \mu \mathrm{m} \mathrm{min}{ }^{-1}$. A major concern of these experiments is the biocompatibility of the UV illumination. To keep the UV intensity and dose in the safety range of the cells, the UV light is irradiated from the bottom of the coating. Azobenzene absorbs the incoming light and forms a light intensity gradient along the film thickness. Therefore, on one hand, the light intensity is sufficient to generate the topographies at the LCN coating surfaces; on the other hand, the light intensity at the coating surface is kept relatively low, and most of the cells remain viable during light illumination for $12 \mathrm{~h}$. Although in their work the UV-light intensity is low, it is still not preferred for biomedical applications. Therefore, in the next section, the use of visible light to generate surface topographies is elaborated on.

\subsection{Visible Light-Activated Surface Topographies}

In the foregoing work, UV light is used to activate surface topographies. Using UV light has a number of disadvantages; for instance, it reduces the lifetime of the coatings due to the degradation of azobenzene molecules, introduces heat that is unsafe, and eventually damages the surrounding environment. Therefore, using visible light to initiate the photo-isomerization process of azobenzene molecules has become more desirable, especially for biomedical applications, as elaborated in section 3.4. A number of approaches to modifying azobenzene have been developed. Yin et al. have designed crosslinked liquid crystal polymers with a longer conjugated azotolane structure as side chains. ${ }^{[63]}$ The absorption band of the azotolane group is shifted toward higher wavelengths by the increase in the $\pi$ orbital energy level and the decrease in the $\pi^{*}$ orbital energy. The authors further demonstrate that by alternating blue light at $430 \mathrm{~nm}$ and green light at $570 \mathrm{~nm}$, this polymer film can bend and relax, respectively.

Alternatively, azobenzene orthosubstituted with methoxy, fluoro-, chloro-, and bromo- groups (Figure 6a) separate the $\mathrm{n}-\pi^{*}$ transitions of the cis and trans isomers, and the absorption band of the lower energy trans isomer is strongly shifted to the visible spectrum. ${ }^{[64,65]}$ Kumar et al. ${ }^{[66]}$ co-polymerized orthofluoroazobenzene, which is further denoted as F-azo, into an LCN by functionalization with reactive end groups (molecule 5 , Figure 6a). The isomerization of F-azo is switched by blue and green light. The F-azo-modified LCNs can be activated under sunlight to give a still-not-well-understood chaotic deformation behavior. Hendrikx et al. ${ }^{[67]}$ further confined the F-azo-modified LCNs to a rigid substrate and studied the corresponding surface topography under irradiation by visible light. In this work, $\approx 10 \mathrm{w} \%$ of the reactive F-azo compound is copolymerized with the cholesteric LCN monomers. Figure 6b shows the top-view of the LCN surfaces; the color bar indicates that the surface becomes more protruded. The initial coating is relatively flat, exhibiting a small roughness below $40 \mathrm{~nm}$. When the coating is exposed to green light at $530 \mathrm{~nm}$ through a zigzag photomask, a surface topography is created in accordance with the pattern information of the mask. Measurements show that surface protrusions of $\approx 150 \mathrm{~nm}$ are generated in a coating with a thickness of $18 \mu \mathrm{m}$. This moderate deformation of less than $1 \%$ of the coating thickness, which is defined as the quotient of height difference and coating thickness, is due to the low LED light intensity of $10-20 \mathrm{~mW} \mathrm{~cm}{ }^{-2}$. As a reference, the surface topographies with the deformation depth of $10 \%$ shown in Figure $2 \mathrm{c}$ are generated by UV light with an intensity of $300 \mathrm{~mW} \mathrm{~cm}{ }^{-2}$, nearly 30 times stronger than that of the green light. When the green-light exposure is stopped the surface topography (a)<smiles>CCC(=O)Nc1cc(Cl)c(N=Nc2c(Cl)cc(NC(=O)CC)cc2Cl)c(Cl)c1</smiles>

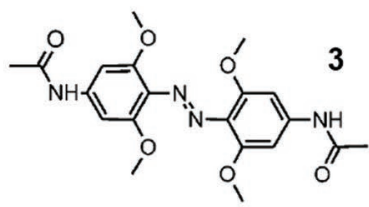

4

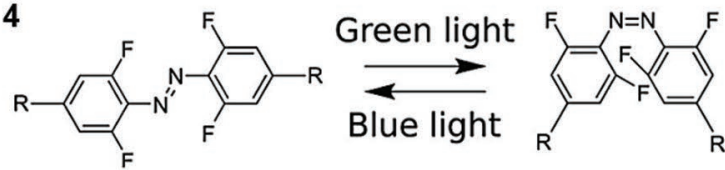<smiles>C=C(C)C(=O)OCCCCCCOC(=O)c1cc(F)c(N=Nc2c(F)cc(C(=O)OCCCCCCOC(=O)C(=C)C)cc2F)c(F)c1</smiles>

5

Figure 6. Visible-light responsive system. a) ortho-substituted azobenzene with 1 bromo-, $\mathbf{2}$ chloro-, $\mathbf{3}$ methoxy-, and $\mathbf{4}$ fluoro- groups. b) The dynamic
surface topographies of LCNs that are created and erased reversibly by illuminating with visible light at different wavelengths. Reproduced with permission. ${ }^{[67]}$ Copyright 2018, Wiley-VCH.

$530 \mathrm{~nm}$ (b)
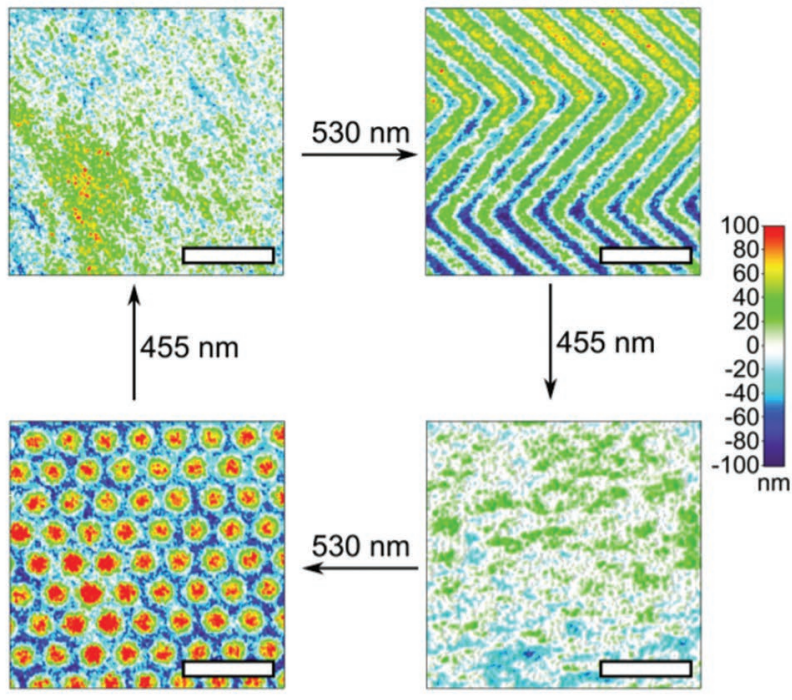
$455 \mathrm{~nm}$

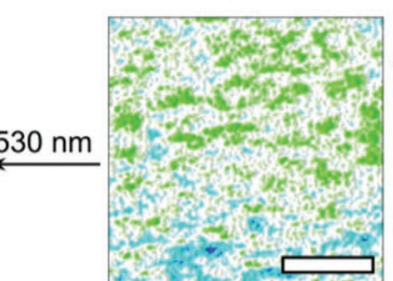

$\mathrm{nm}$ 
remains. It takes $12 \mathrm{~d}$ to relax to $80 \mathrm{~nm}, \approx 50 \%$ of the height directly measured after 30 min illumination. The UV-vis measurement of the thermal relaxation process of F-azo in solution suggests its half-life time in the dark at room temperature is $281 \mathrm{~h}$. These results indicate that the mechanical relaxation is correlated to the chemical process of the cis-to-trans reverse isomerization. It is known that ortho substitution slows down the cis-to-trans thermal relaxation process. A study has shown that the half-life time of the cis isomers of some of the orthosubstituted azobenzene molecules may even extend to 700 h. ${ }^{[68]}$ For comparison, the half-life time of the current unsubstituted azobenzenes is on the order of hours. The back-relaxation of the F-substituted azobenzene cis-to-trans isomer is promoted by blue-light irradiation, by thermally heating up the sample, or a combination of both. In the work of Hendrikx et al., the surface topographies are removed by $455 \mathrm{~nm}$ light illumination with an intensity $<1 \mathrm{~mW} \mathrm{~cm}{ }^{-2}$ at $40^{\circ} \mathrm{C}$ for $23 \mathrm{~min}$. The long illumination period also needed here is attributed to the extremely low light intensity. This flat surface can be further recycled. Using a difference photomask, e.g., circular openings, pillars are created. Using these low light intensities, the processes can be repeated many times. Potentially, this route can be applied for biomedical applications, where the low-intensity visible light is harmless to the various organs and living matter.

\subsection{Active LCN-based cilia}

In the foregoing sections, coatings are entirely confined on substrates. In this section, we will discuss LCN-based motile surfaces consisting of cilia-like dynamic structures. Van Oosten et al. have created artificial cilia on a glass substrate by inkjet printing LCNs. ${ }^{[69]}$ The flat, locally nonadhering filaments change their shape to a curl when addressed by light (see Figure 7a). The splay alignment within the cilia induces bending upon the UV-induced change in the order parameter. There are a number of innovations in the fabrication procedure of cilia. First, the cilia need to bend upward away from the substrate. For this purpose, a homeotropic molecular orientation is needed at the glass side of the cilia, and a planar alignment is consequently needed at the opposite side. This design requires a special fabrication technique whereby the alignment is controlled by means of surfactants. ${ }^{[70]}$ The localized surface release is achieved by using a previously printed polyvinylalcohol structure as the sacrificial layer. The bending proceeds at reasonable speeds in the second region depending on the light intensity. A side view of the cilia is shown in Figure 7a. The bending is actuated when immersed in water, which also demonstrates that we are looking at a pure photochemical effect avoiding the influence of heating. An interesting option that Van Oosten worked out is that the cilia can be made of sections provided with different azobenzene compounds, as illustrated in Figure $7 \mathrm{a}$ by the yellow and red colors. Depending on the wavelength of the actuating light, a different part of the cilium is actuated, and complex motion figures are possible depending on the wavelength sequence used during actuation. This approach is an especially interesting option when the cilia are used to pump water in microfluidic channels, where asymmetric motion figures are needed to prevent backflow.

An alternative technique to form light-responsive cilia at a glass surface has been developed by Gelebart et al.[71] Arrays of LCN cilia are produced by fiber drawing from printed LC monomer droplets on the glass substrate. When the still liquid droplet is brought in contact with another object (needle, glass plate, etc.) that moves away from the surface, the fibers are drawn. The elongational flow controls the alignment of the liquid crystal monomer, which is frozen-in by photopolymerization. To facilitate fiber drawing, the rheology of the LC monomer is adjusted by a thiol addition reaction that forms oligomers of several units of the initial monomer. The light sensitivity is acquired by blending an azobenzene diacrylate molecule, as discussed above. The molecular alignment along the long axis of the fibers is maintained within the time frame of the drawing process and is permanently fixed by photopolymerization, thereby forming a network with a relatively low crosslink density. Having many droplets side by side, an array of fibers is formed. When the fiber arrays are illuminated with UV light from the side, they bend toward light source as the absorption of azobenzene creates a gradient in the light intensity.

This cluster of bending fibers is further investigated for material transport. The bending direction of the fiber is in the direction of the light source and relies on intensity gradients throughout the cross-section of the fibers. By rotating the light source, the fibers are brought into a rotating motion, which can be made asymmetric by controlling the movement of the light.
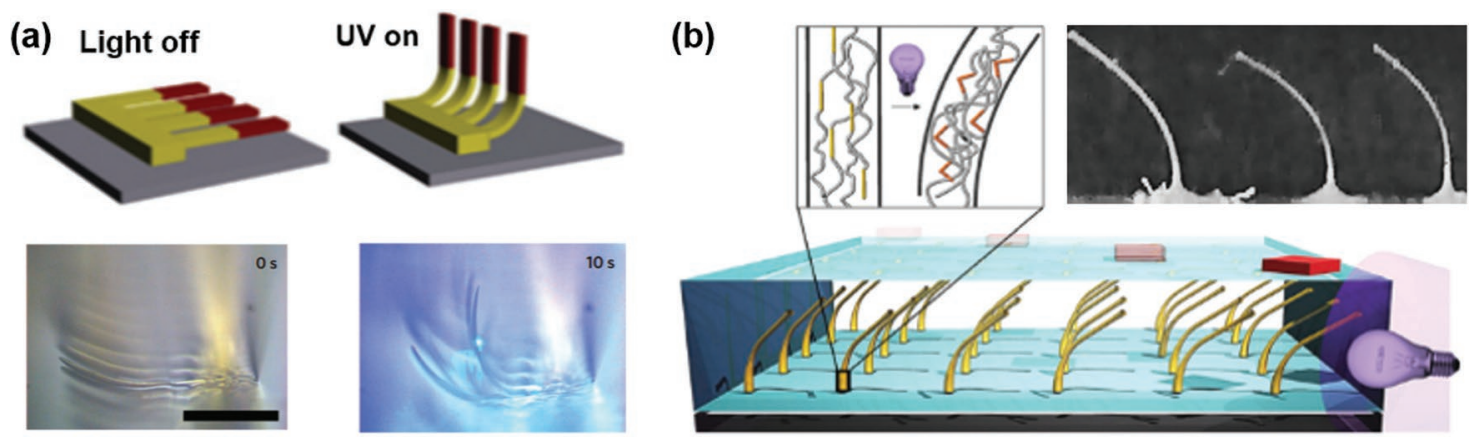

Figure 7. Surface-integrated photoactive structures. a) Artificial cilia that curl with exposure to UV light. Reproduced with permission. ${ }^{699}$ Copyright 2009, Springer Nature. b) Large-area fabricated fiber arrays bend toward the UV light. Reproduced with permission. ${ }^{[71]}$ Copyright 2016, Wiley-VCH. 
When immersed in a relatively low-viscous silicone oil, the mass transport of the oil can also transport polymer platelets floating on top of the liquid.

\subsection{Light-Induced Thermal Effect}

Irradiating soft matter with light can be associated with heating, for example, dye molecules, such as azobenzene, that generate a photomechanical effect based on isomerization chemistry also convert absorbed light energy into heat. Therefore, some warming of the exposed area of the sample and heat effects are not avoidable. As described above, Van Oosten immerses LCNs in water during actuation to avoid heating. This has been further elaborated by Vantomme et al. ${ }^{[72]}$ who compare the temperature effects of bending LCNs in air and in water. Liu et al. ${ }^{[24]}$ have replaced azobenzene molecules with an inert dye, which after some adjustment of the concentration, exhibits the same absorbance as azobenzene. As it comes out, the thermal effect in free-standing films is considerable, but it is less in the LCN coating, although not completely ignorable. Evidently, the contact with the substrate reduces the heating effect. The formation of protrusions by thermal heating is estimated using thermal expansion calculations that use 1D expansion based on known volume expansion figures and the measured data from an infrared camera. ${ }^{[24]}$ Additionally, here, the conclusion is that thermal expansion plays a role, but it does not explain fully the total height of the structures formed.

Several groups ${ }^{[38,73]}$ have reported on the reduction of the elastic modulus of azobenzene-containing linear polymers during light illumination, which is referred to as "photofluidization," "photosoftening," and "photoplasticization." These studies are mainly based on linear polymers where the chains can flow easily. Therefore, mass displacement or material transport has been demonstrated in some cases. White et al. have reported on densely crosslinked liquid crystal networks modified with $20 \%$ azobenzene, and the modulus can be reduced by one to two orders of magnitude upon exposure to $442 \mathrm{~nm}$ polarized light. ${ }^{[74,75]}$ Kumar et al. have further built on this work by reducing the azobenzene concentration to $2 \%$ to minimize the light-induced heating. ${ }^{[6]}$ In their experiments, the LCN is measured as $1.8 \mathrm{GPa}$ in the dark. When exposed to a UV LED light source, the modulus drops to $1 \mathrm{GPa}$. By switching off the light, the initially higher modulus is recovered within $1 \mathrm{~min}$. The authors further include blue light during the UV exposure. This experiment was designed under the observation that the oscillation dynamics of the trans-cis isomerization of azobenzene promotes the formation of free volume in LCNs. ${ }^{[26]}$ Under these conditions, the modulus decreases further until it reaches the rubbery plateau with a modulus of $10 \mathrm{MPa}$. When the light stops, the modulus directly recovers back to its initial value. The temperature of the sample was recorded during light illumination, which might partly also explain the reduction in modulus. However, a control experiment with temperature only reveals the modulus-softening influence of the light sources. The underlying mechanism for the light-induced plasticization is attributed to the oscillating strain of the network. The photosoftening also explains why a large free volume of $\approx 10 \%$ and the corresponding surface topography created in a glassy polymer network take place without fatigue.

\section{Conclusions and Outlook}

The morphing of free-standing LCNs has already been demonstrated for several decades. However, due to their soft elastic nature, LCN-based films exert a limited force to perform work, they respond relatively slow and are less accurate than traditional hard materials. One way to approach this problem is to make the film undergo a self-reinforcing deformation by addressing the order of the mesogens around an array of topological defects. ${ }^{[77]}$ Another approach to address this problem is to confine $3 \mathrm{D}$ deformation in $2 \mathrm{D}$ composites by strongly adhering the film to a solid substrate, and this action manifests itself as dynamic surface topographies. From these topographical deformations of solid coatings, new functions may evolve including adaptive tribology, friction and adhesion, dynamic wettability, and the manipulation of materials and living matter brought on top of the coating. A unique example can be found in haptic applications, where tactile feedback to the human user is given during human-machine interaction. The most common existing examples are mobile phones and tablets, where the whole device vibrates when one touches the screen, in other words, the haptic feedback is global, rather than local. In addition to visual changes, a local response is obtained when, upon touching a surface, the device provides mechanical information only at the location of the fingertip by a (oscillatory) deformation or a change in the friction coefficient. This can help visually impaired people. However, it can also support typing on a tablet screen with a projected keyboard. Other typical applications include tactile information transfer to a doctor during remote surgery; the addition of sensitivity, touch, and adjustable friction to robotic arms; and the addition of complexity to video games. The presented materials are a first important step toward new soft robotic functions. However, new material designs are necessary, as is their embedment in fully operating systems where sensing and acting are brought in close loops within the same micromachine or even within the same material.

The LCN technology that we describe here can be easily integrated into applications through existing fabrication processes that are established in the LCD industry. Many of the materials that are discussed in this chapter are commercially available and are already widely applied in many commercial display panels.

\section{Acknowledgements}

The results presented are part of research programmes financed by Guangdong Innovative Research Team Program (No. 2013C102), European Research Commission under ERC Advanced Grant 66999 (VIBRATE), and NWO VENI grant 15135, and additional information is available from the author.

\section{Conflict of Interest}

The authors declare no conflict of interest. 


\section{Keywords}

dynamic surface topographies, light responsive materials, liquid crystal networks

Received: February 12, 2019

Revised: March 28, 2019

Published online: April 29, 2019

[1] J. Y. Lim, H. J. Donahue, Tissue Eng. 2007, 13, 1879

[2] B. N. J. Persson, O. Albohr, U. Tartaglino, A. I. Volokitin, E. Tosatti, J. Phys.: Condens. Matter 2005, 17, R1.

[3] C. Masaki, G. B. Schneider, R. Zaharias, D. Seabold, C. Stanford, Clin. Oral Implants Res. 2005, 16, 650

[4] H. Culbertson, S. B. Schorr, A. M. Okamura, Annu. Rev. Control Robotics Auton. Syst. 2018, 1, 385.

[5] L. Skedung, M. Arvidsson, J. Y. Chung, C. M. Stafford, B. Berglund, M. W. Rutland, Sci. Rep. 2013, 3, 2617.

[6] Y. Bar-Cohen, IJASS 2012, 13, 1.

[7] T. M. de Jong, D. K. G. de Boer, C. W. M. Bastiaansen, Opt. Express 2011, 19, 15127.

[8] H. Schift, M. Halbeisen, U. Schütz, B. Delahoche, K. Vogelsang, J. Gobrecht, Microelectron. Eng. 2006, 83, 855.

[9] M. Ibn-Elhaj, M. Schadt, Nature 2001, 410, 796.

[10] D. J. Broer, R. A. M. Hikmet, G. Challa, Makromol. Chem. 1989, 190, 3201.

[11] D. J. Broer, J. Boven, G. N. Mol, G. Challa, Makromol. Chem. 1989, 190, 2255

[12] P. Keller, D. L. Thomsen, M.-H. Li, Marcomol. 2002, 35, 581.

[13] D. J. Broer, G. P. C. Crawford, S. Zumer (Eds), Cross-Linked Liquid Crystalline Systems: From Rigid Polymer Networks to Elastomers, 1st Edition, Liquid Crystals Book Series, CRC Press, Boca Raton, FL 2011, Chapter 1.

[14] P. G. de Gennes, J. Prost, The Physics of Liquid Crystals, 2nd ed., Clarendon, Oxford, UK 1993.

[15] D. J. Broer, J. Lub, G. N. Mol, Nature 1995, 378, 467.

[16] D. J. Broer, I. Heynderickx, Macromol. 1990, 23, 2474.

[17] J. Lub, D. J. Broer, R. A. M. Hikmet, K. G. J. Nierop, Liq. Cryst. 1995, $18,319$.

[18] D. J. Broer, G. N. Mol, Polym. Eng. Sci. 1991, 31, 625

[19] R. A. M. Hikmet, B. H. Zwerver, D. J. Broer, Polymer 1992, 33, 89.

[20] G. N. Mol, K. D. Harris, C. W. M. Bastiaansen, D. J. Broer, Adv. Funct. Mater. 2005, 15, 1155.

[21] T. Seki, D. Yamaoka, T. Takeshima, Y. Nagashima, M. Hara, S. Nagano, Mol. Cryst. Liq. Cryst. 2017, 644, 52.

[22] T. Seki, S. Nagano, Chem. Lett. 2008, 37, 484.

[23] T. Seki, T. Tamaki, Chem. Lett. 1993, 22, 1739.

[24] D. Liu, C. W. M. Bastiaansen, J. M. J. den Toonder, D. J. Broer, Macromol. 2012, 45, 8005.

[25] O. M. Tanchak, C. J. Barrett, Macromol. 2005, 38, 10566

[26] D. Liu, D. J. Broer, Nat. Commun. 2015, 6, 8334.

[27] Z. Cheng, S. Ma, Y. Zhang, S. Huang, Y. Chen, H. Yu, Macromol. 2017, 20, 8317.

[28] S. Ma, X. Li, S. Huang, J. Hu, H. Yu, Angew. Chem. 2018, 57, 12524.

[29] H. Yu, T. Ikeda, Adv. Mater. 2011, 23, 2149.

[30] C. L. Van Oosten, C. W. M. Bastiaansen, D. J. Broer, Nat. Mater. 2009, 8, 677

[31] M. Camacho-Lopez, H. Finkelmann, P. Palffy-Muhoray, M. Shelley, Nat. Mater. 2004, 3, 307.

[32] S. Iamsaard, S. J. Aßhoff, B. Matt, T. Kudernac, J. J. L. M. Cornelissen, S. P. Fletcher, N. Katsonis, Nat. Chem. 2014, 6, 226.

[33] L. Yu, H. Yu, Q. Li, Nanoscience with Liquid Crystals (Ed.: Q. Li) Springer,Berlin, 2014, p. 301.
[34] M Lahikainen, H. Zeng, A. Priimagi, Nat. Commun. 2018, 9, 4148.

[35] Y. Yu, M. Nakano, T. Ikeda, Nature 2003, 425, 145.

[36] T. Kosa, L. Sukhomlinova, L. Su, B. Taheri, T. J. White, T. J. Bunning, Nature 2012, 485, 347.

[37] D. Liu, Liq. Cryst. 2016, 43, 2136.

[38] G. Fang, J. Maclennan, Y. Yi, M. Glaser, M. Farrow, E. Korblova, D. Walba, T. Furtak, N. Clark, Nat. Commun. 2013, 4, 1521

[39] M. Hendrikx, B. Sırma, A. P. H. J. Schenning, D. Liu, D. J. Broer, Adv. Mater. Interfaces 2018, 5, 1800810.

[40] M. E. Sousa, D. J. Broer, C. W. M. Bastiaansen, L. B. Freund, G. P. Crawford, Adv. Mater. 2006, 18, 1842.

[41] G. Babakhanova, T. Turiv, Y. Guo, M. Hendrikx, Q. Wei, A. P. H. J. Schenning, D. J. Broer, O. D. Lavrentovich, Nat. Commun. 2018, 9, 456

[42] K. D. Harris, R. Cuypers, P. Scheibe, C. L. van Oosten, C. W. M. Bastiaansen, J. Lub, D. J. Broer, J. Mater. Chem. 2005, 15, 5043.

[43] C. L. van Oosten, K. D. Harris, C. W. M. Bastiaansen, D. J. Broer, Eur. Phys. J. 2007, 23, 329.

[44] D. Liu, D. J. Broer, Angew. Chem., Int. Ed. Engl. 2014, 126, 4630.

[45] D. Liu, L. Liu, P. R. Onck, D. J. Broer, Proc. Natl. Acad. Sci. USA 2015, 112, 3880

[46] W. Shizhu, H. Ping, Principles of Tribology, Tsinghua University Press, Tsinghua, 2012.

[47] D. Liu, D. J. Broer, Soft Mater 2014, 10, 7952.

[48] S. Tang, Master Thesis, Wettability in liquid crystal coatings based on switchable surface topographies, Eindhoven University of Technology, Eindhoven, The Netherlands 2015.

[49] C. Li, Y. Zhang, J. Ju, F. Cheng, M. Liu, L. Jiang, Y. Yu, Adv. Funct. Mater. 2012, 22, 760.

[50] R. Eelkema, M. M. Pollard, ]. Vicario, N. Katsonis, B. S. Ramon, C. W. M. Bastiaansen, D. J. Broer, B. L Feringa, Nature 2006, 440, 163.

[51] A. Bosco, M. G. M. Jogejan, R. Eelkema, N. Katsonis, E. Lacaze, A. Ferrarini, B. L. Feringa, J. Am. Chem. Soc. 2008, 130, 14615.

[52] A. Ryabchun, A. Bobrovsky, J. Stumpe, V. Shiaev, Adv. Opt. Mater. 2015, 3, 1273.

[53] G. Babakhanova, International Liquid Crystal Conference 2018, Kyoto, Japan, P1-C2-3.

[54] M. Radtke, A. Patzelt, F. Knorr, J. Lademann, R. R. Netz, Eur. J. Pharm. Biopharm. 2017, 116, 125.

[55] W. Feng, D. J. Broer, D. Liu, Adv. Mater. 2018, 30, 1704970.

[56] A. Sayyah, M. N. Horenstein, M. K. Mazumder, G. Ahmadi, J. Electrostat. 2016, 81, 24

[57] A. Sayyah, D. R. Crowell, A. Raychowdhury, M. N. Horenstein, M. K. Mazumder, J. Electrostat. 2017, 87, 173.

[58] S. Zhou, A. Sokolov, O. D. Lavrentovich, I. S. Aranson, Proc. Natl. Acad. Sci. USA 2014, 111, 1265.

[59] P. C. Mushenheim, R. R. Trivedi, S. S. Roy, M. S. Arnold, D. B. Weibelb, N. L. Abbott, Soft Matter 2015, 11, 6821.

[60] O. D Lavrentovich, Curr. Opin. Colloid Interface Sci. 2016, 21, 97.

[61] C. Kocer, J. ter Schiphorst, M. Hendrikx, H. G. Kassa, P. Leclere, A. P. H. J. Schenning, P. Jonkheijm, Adv. Mater. 2017, 29, 1606407.

[62] M. J. Balby, N. Gadegaard, R. Tare, A. Andar, M. O. Riehle, P. Herzyk, C. D. W. Wilkinson, R. O. C. Oreffo, Nat. Mater. 2017, $13,558$.

[63] R. Yin, W. Xu, M. Kondo, C. Yen, J. Mamiya, T. Ikeda, Y. Yu, J. Mater. Chem. 2009, 19, 3141.

[64] S. Samanta, A. A. Beharry, O. Sadovski, T. M. McCormick, A. Babalhavaeji, V. Tropepe, G. A. Woolley, J. Am. Chem. Soc. 2013, 135, 9777.

[65] M. Y. Paik, S. Krishnan, F. You, X. Li, A. Hexemer, Y. Ando, S. H. Kang, D. A. Fischer, E. J. Kramer, C. K. Ober, Langmuir 2007, 23, 5110.

[66] K. Kumar, C. Knie, D. Bléger, M. A. Peletier, H. Friedrich, S. Hecht, D. J Broer, M. G. Debije, A. P. H. J. Schenning, Nat. Commun. 2016, 7, 11975 
[67] M. Hendrikx, J. ter Schiphorst, E. P. A. van Heeswijk, G. Koçer, C. Knie, D. Bléger, S. Hecht, P. Jonkheijm, D. J Broer, A. P. H. J. Schenning, Small 2018, 14, 1803274.

[68] D. Bleger, J. Schwarz, A. M. Brouwer, S. Hecht, J. Am. Chem. Soc. 2012, 134, 20597.

[69] C. L. van Oosten, C. W. M. Bastiaansen, D. J. Broer, Nat. Mater. 2009, 8, 667 .

[70] A. J. J. Kragt, D. J. Broer, A. P. H. J. Schenning, Adv. Funct. Mater. 2018, 28, 1704756

[71] A. H. Gelebart, M. Mc Bride, A. P. H. J. Schenning, C. N. Bowman, D. J. Broer, Adv. Funct. Mater. 2016, 26, 5322.
[72] G. Vantomme, A. H. Gelebart, D. J. Broer, E. W. Meijer, J. Polym. Sci. A: Polym. Chem. 2018, 56, 1331.

[73] J. M. Harrison, D. Goldbaum, T. C. Corkery, C. I. Barrett, R. R. Chromik, J. Mater. Chem. C 2015, 3, 995.

[74] K. M. Lee, T. J. White, Macromol. 2012, 45, 7163.

[75] K. M. Lee, H. Koerner, R. A. Vaia, T. J. Bunninga, T. J. White, Soft Matter 2011, 7, 4318.

[76] K. Kumar, A. P. H. J. Schenning, D. J. Broer, D. Liu, Soft Matter 2016, 12, 3196

[77] T. H. Ware, M. E. McConney, J. J. Wie, V. P. Tondiglia, T. J. White, Science 2015, 347, 982 